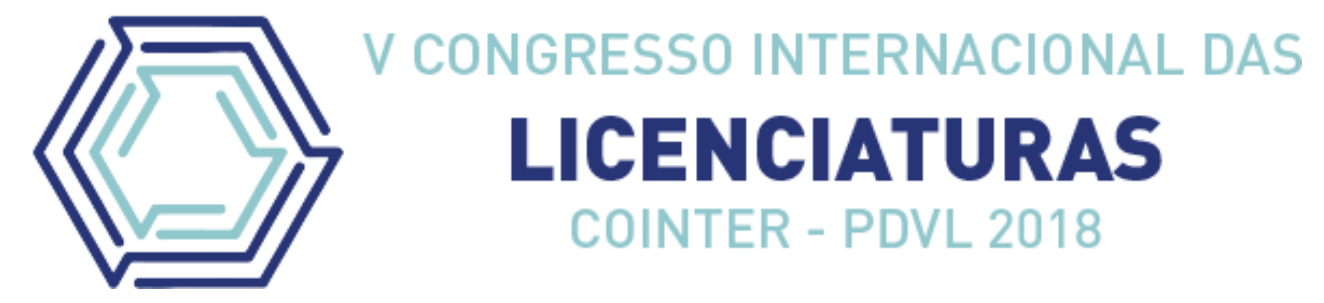

\title{
COMO PREPARAR OS PROFESSORES DA EDUCAÇÃO INFANTIL PARA A ESCOLARIZAÇÃO DA CRIANÇA COM MICROCEFALIA
}

\section{HOW TO PREPARE TEACHERS OF CHILD EDUCATION FOR SCHOOLING OF CHILDREN WITH MICROCEPHAL}

\author{
Apresentação: Comunicação Oral \\ Íris Regina Felix Martins de Melo Jaqueline Costa da Silva Lima ${ }^{2}$; Hander de Lucena \\ Neri $^{3}$; Islane Cristina Martins ${ }^{4}$
}

DOI: $\underline{\text { https://doi.org/10.31692/2358-9728.VCOINTERPDVL.2018.00047 }}$

\section{Resumo:}

O presente trabalho tem como objetivo desenvolver estratégias de ensino-aprendizagem que se adapte ás necessidades especiais de crianças portadoras de microcefalia dando o suporte pedagógico necessário aos professores da educação Infantil que contribuem para a escolarização das mesmas, através da formação continuada e de materiais pedagógicos que promovam assim uma aprendizagem inclusiva que seja significativa, conseguindo transmitir aos alunos conhecimentos, habilidades e valores capazes de aprimorar o seu desenvolvimento social, cognitivo e afetivo. Foi feito um levantamento da literatura em Março de 2018, na base de dados Google Acadêmico. Os descritores utilizados foram "Microcefalia" AND "Educação Infantil" AND "Educação Inclusiva". Foram selecionados 08 artigos sendo incluídos segundo os critérios de elegibilidade. Os critérios de inclusão foram: artigos nos idiomas inglês, espanhol e português, que analisassem se os professores na modalidade da Educação Infantil estão preparados para receber estas crianças na faixa etária de 04 - 06 anos na rede pública. Os critérios de exclusão foram artigos de revisão de literatura ou metanálise. Como resultado, foi possível considerar que os fundamentos teóricos metodológicos foram agrupados por semelhanças de informações no tocante à escolarização de crianças com necessidades especiais como também da inclusão escolar das mesmas, sendo embasados pela Lei Brasileira de Inclusão (Lei ${ }^{\circ}$ 13.146). Os textos apontam como acontece o aprendizado das crianças que estão neste contexto, as reais necessidades escolares e estratégias pedagógicas que possibilite a aprendizagem. Nos deparamos com a necessidade de formação específica para que os profissionais tenham suporte e conhecimento teórico para trabalharem com as especificidades das crianças. Em conclusão, para que os alunos com microcefalia possam ser englobados de forma eficaz, os educadores devem compreender a anomalia e desenvolver atividades que estimulem e/ou reabilitem os campos afetados como o neurológico, psíquico, motor, cognitivo e físico através de atividades específicas e peculiares

Palavras-chave: Educação inclusiva, Crianças com microcefalia, Apoio pedagógico.

\footnotetext{
${ }^{1}$ Especialista em Neuropedagogia, UFPE, irisneuroeduc@gmail.com

${ }^{2}$ Letras Libras, Uniasselvi, jackeline.libras@gmail.com

${ }^{3}$ Pedagogia, FCE, patylucena80@ hotmail.com

${ }^{4}$ Doutoranda em Neurociências, POSNEURO/UFPE, islanemartins@gmail.com
} 



\begin{abstract}
The present work aims to develop teaching-learning strategies that adapt to the special needs of children with microcephaly giving the necessary pedagogical support to the teachers of Infant education that contribute to the schooling of the same, through the continuous training and pedagogical materials that thus promoting an inclusive learning that is meaningful and able to transmit to the students knowledge, skills and values capable of improving their social, cognitive and affective development. A survey of the literature was made in March 2018 in the Google Scholar database. The descriptors used were "Microcephaly" AND "Infant Education" AND "Inclusive Education". Eight articles were selected according to the eligibility criteria. The inclusion criteria were: articles in the English, Spanish and Portuguese languages, which analyzed whether the teachers in the Child Education modality are prepared to receive these children in the age group of 04 - 06 years in the public network. Exclusion criteria were articles of literature review or meta-analysis. As a result, it was possible to consider that the theoretical methodological foundations were grouped by similarities of information regarding the schooling of children with special needs as well as the school inclusion of the same, being supported by the Brazilian Inclusion Law (Law $n^{\circ} 13.146$ ). The texts point out how the children's learning in this context happens, the real school needs and pedagogical strategies that make learning possible. We are faced with the need for specific training so that professionals have the support and theoretical knowledge to work with the specifics of children. In conclusion, in order for students with microcephaly to be effectively encompassed, educators must understand the anomaly and develop activities that stimulate and / or rehabilitate affected fields such as neurological, psychic, motor, cognitive and physical through specific and peculiar activitie.
\end{abstract}

Keywords: Inclusive education, Children with microcephaly, Pedagogical support.

\title{
Introdução
}

A ocorrência de casos confirmados em Pernambuco de crianças com microcefalia advinda da Síndrome Congênita do Zika Vírus tem mostrado ao campo educacional a necessidade de maior compreensão sobre esta condição neurológica bem como o conhecimento necessário para a efetivação da inclusão de crianças portadoras de necessidades especiais.

A grande dificuldade apresentada inicialmente foi a falta de informações sobre o assunto e como se daria a inclusão no espaço, materiais e métodos educacionais adequados para este novo cliente. A criança chegou à creche e foi muito bem assistida por todos os profissionais, em especial por sua professora e cuidadora.

Microcefalia é uma desordem neurológica, em que a circunferência da cabeça é menor do que a média para a idade e sexo da criança. A pessoa com essa deficiência na 
maioria das vezes apresenta dificuldades ou nítido atraso em seu desenvolvimento neuropsicomotor, aquisição da fala e outras habilidades. (MARCELLI; COHEN, 2010).

A evidência crucial para determinar a ligação da microcefalia com o vírus da ZIKAV foi um teste feito no Instituto Evandro Chagas, órgão vinculado ao Ministério Público do Pará, que detectou a presença do vírus ZIKAV em amostras de sangue coletadas de um bebê que nasceu com microcefalia no Ceará e acabou morrendo. (FIOCRUZ, Fundação Oswaldo Cruz. Especiais ZIKAV, 2015).

É possível a utilização de vários materiais que auxiliem a desenvolver o processo de ensino e de aprendizagem, isso faz com que facilite a relação professor aluno - conhecimento. O uso de materiais e métodos adequados é muito importante para uma melhor aplicação dos conteúdos propondo maior interação e profundidade nos indivíduos participantes do processo de ensino-aprendizagem.

A relevância e importância deste trabalho surgem em virtude da preocupação do processo de escolarização das crianças que nasceram com a Síndrome Congênita do Vírus da ZIKAV visto que a Lei ${ }^{\circ} 13.146$ - Lei Brasileira de Inclusão assegura sua inserção no âmbito escolar e da necessidade de conhecimentos específicos para a realização do trabalho pedagógico. Portanto, é imprescindível que busquemos conhecimentos facilitadores para a prática educacional através de estudos e no desenvolvimento de métodos e materiais que tornem a aprendizagem mais dinâmica e eficaz para que tais recursos alcancem os objetivos propostos. Ainda, pelo fato da grande demanda de crianças com microcefalia e do acolhimento de uma dessas na creche (em Bezerros- PE no ano vigente) tendo relação direta com ZIKAV. Tal criança tem dois anos e oito meses foi matriculada na referida creche. Para a diretora da instituição foi uma surpresa e motivo de preocupação visto que não estavam preparadas em termos de conhecimento para lidar com essa nova demanda de alunos.

\section{Fundamentação Teórica}

Aprofundar nossos conhecimentos a respeito da inclusão de crianças com necessidades especiais faz parte do papel do pedagogo como um ser pesquisador, pois segundo Libâneo este profissional é um criador de caminhos para se chegar a um fim, podendo então lhe dar com qualquer que seja o tipo de educação que lhe esteja sendo apresentada, bem como utilizador de métodos educacionais que facilitem a aprendizagem do aluno. 
A inclusão escolar de crianças com necessidades educacionais especiais é um tema de grande relevância e vem ganhando espaço cada vez maior em debates e discussões que explicitam a necessidade da escola em atender às diferenças intrínsecas à condição humana. (LEI)

Para Vygotsky (1987), há potencialidade e capacidade nas pessoas com deficiência, mas entende que, para poder desenvolvê-las, devem ser lhes oferecidas condições materiais e instrumentais adequadas. Com isso, deve-se oferecer a tais pessoas uma educação que lhes oportunize a apropriação da cultura histórica e socialmente construída, para melhores possibilidades de desenvolvimento.

Aprendizagem é a maneira pela qual os valores, comportamentos e conhecimentos são alcançados ou alterados, como produto de formação e experiências. Esta relação pode ser examinada de diferentes maneiras, levando -se em consideração diferentes teorias de aprendizagem. (VELÁSQUEZ, 2001). "O aprendizado é mais do que a aquisição de capacidade para pensar; é a aquisição de muitas capacidades especializadas para pensar sobre várias coisas." (VYGOTSKY, 1991, p.55)

Quando a criança tem microcefalia pode apresentar atraso mental, alterações físicas como dificuldade para andar, problemas de fala e hiperatividade ou convulsões, por exemplo. Porém, estas consequências da doença não surgem em todos os casos e, algumas crianças podem se desenvolver normalmente e ter uma inteligência normal porque isso depende da gravidade da sua microcefalia.

A pessoa com essa deficiência na maioria das vezes apresenta dificuldades ou nítido atraso em seu desenvolvimento neuropsicomotor, aquisição da fala e outras habilidades (comportamento adaptativo conceptuais, sociais ou práticos) (MARCELLI; COHEN, 2010).

Portanto, percebe-se que as crianças com microcefalia necessitam de atenção e cuidados específicos. A inclusão escolar do deficiente múltiplo - pessoas com duas ou mais deficiências de base associada - que, na maioria das vezes, é percebido como o educando com necessidades educacionais "mais acentuadas", é fato bastante recente na educação brasileira (MEC, 2002).

Para Silveira (2006), Nos questionamentos sobre a possibilidade de inclusão escolar dessa população, ainda habitam no imaginário social e individual dos profissionais da educação e dos familiares dessas crianças, desconhecimento e 
dúvidas que culminam em incertezas sobre os benefícios e as possibilidades da inclusão.

É consenso que a pessoa com necessidades educacionais especiais se beneficia das interações sociais e da cultura na qual está inserida, sendo que essas interações, se desenvolvidas de maneira adequada, serão propulsoras de mediações e conflitos necessários ao desenvolvimento pleno do indivíduo e à construção dos processos mentais superiores (Vygotsky, 1987).

Para Vygotsky, a transformação dos processos mentais elementares em funções superiores ocorre por meio das atividades mediadas e por meio das ferramentas psicológicas, o que implica, para esse autor, que a formação da subjetividade individual decorre do relacionamento com os outros.

Desta forma, a inclusão escolar pressupõe mudanças físicas relacionadas a posturas frente às concepções que coabitam na escola, sendo que um dos embates de maior significância é o que se refere à formação de professores em níveis teóricos, práticos e pessoais, que, na maioria das vezes, se mostra bastante insólita para edificar práticas que realmente estimulem a autonomia, a criatividade e a ampliação das competências do aluno com deficiência múltipla.

Para Esteban (1989, p.77), “a concepção que o professor tem de mundo e de homem tem relação com sua concepção sobre o processo de alfabetização, assim como a leitura que faz do desenvolvimento da criança tem relação com a qualidade da sua intervenção".

Por se reconhecer a importância da escola e da família no desenvolvimento das crianças com deficiência múltipla, justifica-se a relevância do presente trabalho. As concepções das pessoas envolvidas no cotidiano dos deficientes múltiplos nos permitem entender a natureza e a qualidade de suas intervenções, considerando que as ações são orientadas pelas concepções historicamente construídas (Oliveira, 1999).

\section{Importância de adaptar os métodos de ensino para crianças com microcefalia}

Analisar o processo de inclusão social de pessoas com deficiência não é uma tarefa fácil, mas vem sendo melhor explorada e tem ganhado espaço em debates sobre a educação na atualidade. A inclusão é vista como um processo de extensão social que vem produzindo uma melhor aproximação e adaptação, 
convocando então todas as pessoas para a construção de uma sociedade sem preconceitos, e para que ofereça oportunidades para esses cidadãos que possuem essa diferença. Uma dificuldade quanto à inclusão é o fator emocional dos alunos, colegas e professores, quanto ao desconhecimento da doença.

O desconhecimento das características dos quadros de doença e deficiência mental, a angústia gerada pelo contato com a deficiência, as imprecisões da etiologia destas doenças ou deficiência ou a inconstância de um mesmo padrão comportamental nestes alunos mobiliza sentimentos que vão do temor ao apego maternal, da raiva gerada pela impotência à negação das possibilidades da intervenção pedagógica. (DOCUMENTO SUBSIDIÁRIO À POLÍTICA DE INCLUSÃO, 2005, p 32)

Percebe-se que as crianças com microcefalia necessitam de atenção e cuidados específicos que de acordo com Fonseca (1995):

(...) nos nossos dias, o direito de ser diferente é também visto como um direito humano, que passa naturalmente pela análise crítica dos critérios sociais que impõe a reprodução e preservação de uma sociedade (e de uma escola) baseada na lógica da homogeneidade em normas de rentabilidade e eficácia, que tendem facilmente a marginalizar e a segregar quem não acompanha as exigências e os ritmos sofisticados.

Precisamos possibilitar instrumentos e métodos de ensino que oportunizem à essas crianças diminuir a distância que suas impossibilidades os permitem para colocarmos o mais próximo possível do desenvolvimento pleno da aprendizagem, estando atentos às suas peculiaridades, desta forma traremos para o "centro" no sentido de dar a elas a devida importância no planejamento educacional formal que têm direito como todas as outras que não tem deficiência.

\section{Metodologia}

Foi feito um levantamento da literatura em Março de 2018, na base de dados Google Acadêmico. Os descritores utilizados foram "Microcefalia" AND "Educação Infantil” AND "Educação Inclusiva”. Foram selecionados 08 artigos sendo incluídos segundo os critérios de elegibilidade. Os critérios de inclusão foram: artigos nos idiomas inglês, espanhol e português, que analisassem se os professores na modalidade da Educação Infantil estão preparados para receber estas crianças na faixa etária de 04 06 anos na rede pública. Os critérios de exclusão foram artigos de revisão de literatura ou metanálise.

\section{Resultados e Discussão}


Com as análises Tabela 1, foi possível considerar que os fundamentos teóricos metodológicos foram agrupados por semelhanças de informações no tocante à escolarização de crianças com necessidades especiais como também da inclusão escolar das mesmas, sendo embasados pela Lei Brasileira de Inclusão (Lei n ${ }^{\circ}$ 13.146). Os textos apontam como acontece o aprendizado das crianças que estão neste contexto, as reais necessidades escolares e estratégias pedagógicas que possibilite a aprendizagem. Nos deparamos com a necessidade de formação específica para que os profissionais tenham suporte e conhecimento teórico para trabalharem com as especificidades das crianças. 
Tabela 1 - Demonstrativo dos artigos que integram a Revisão Integrativa

\begin{tabular}{|c|c|c|c|c|c|c|}
\hline $\mathbf{N}$ & Data & Título & Autores & Periódico & Participantes & Objetivos \\
\hline 1 & 2008 & $\begin{array}{l}\text { Inclusão escolar: } \\
\text { relações entre o } \\
\text { discurso e a pática }\end{array}$ & $\begin{array}{c}\text { Maria Cecília } \\
\text { Pereira Alexandre }\end{array}$ & Monografia & $\begin{array}{l}\text { Uma profissional da } \\
\text { secretaria de educação e } \\
\text { uma profissional da } \\
\text { educação. }\end{array}$ & $\begin{array}{l}\text { Relacionar os fundamentos } \\
\text { teóricos, com o discurso da } \\
\text { professora de turma inclusiva } \\
\text { e sua prática em sala de aula. }\end{array}$ \\
\hline 2 & 2010 & $\begin{array}{l}\text { A escola e o } \\
\text { professor frente ao } \\
\text { currículo inclusivo }\end{array}$ & $\begin{array}{c}\text { Vanessa Caroline } \\
\text { da Silva }\end{array}$ & Monografia & $\begin{array}{l}20 \text { professoras atuantes } \\
\text { nos anos iniciais do } \\
\text { ensino fundamental e que } \\
\text { possuíam alunos com } \\
\text { necessidades } \\
\text { educacionais especiais } \\
\text { em } 2009 \text { da rede pública } \\
\text { de ensino. }\end{array}$ & $\begin{array}{l}\text { Aprofundar estudos sobre o } \\
\text { currículo escolar na } \\
\text { perspectiva inclusiva; } \\
\text { Analisar a contribuição e } \\
\text { organização do mesmo para } \\
\text { o processo de inclusão } \\
\text { escolar de estudantes com } \\
\text { necessidades educacionais } \\
\text { especiais que frequentam os } \\
\text { anos iniciais do ensino } \\
\text { fundamental. }\end{array}$ \\
\hline 4 & 2011 & $\begin{array}{l}\text { Uma criança com } \\
\text { desenvolvimento } \\
\text { atípico: momentos e } \\
\text { contextos de } \\
\text { aprendizagens. }\end{array}$ & $\begin{array}{l}\text { Mariana Xavier } \\
\text { Pereira }\end{array}$ & Monografia & $\begin{array}{l}\text { Familiares, professores e } \\
\text { o aluno João Pedro. }\end{array}$ & $\begin{array}{l}\text { Compreender aspectos do } \\
\text { processo de aprendizagem de } \\
\text { uma criança com } \\
\text { desenvolvimento atípico, } \\
\text { relacionado ao quadro de } \\
\text { sequelas do quadro } \\
\text { neurológico. }\end{array}$ \\
\hline
\end{tabular}




\begin{tabular}{|c|c|c|c|c|c|c|}
\hline $\mathbf{N}$ & Data & Título & Autores & Periódico & Participantes & Objetivos \\
\hline 5 & 2012 & $\begin{array}{c}\text { Nas tramas da } \\
\text { educação inclusiva: } \\
\text { considerações sobre } \\
\text { as políticas públicas } \\
\text { educacionais para a } \\
\text { inclusão }\end{array}$ & $\begin{array}{c}\text { Priscila } \\
\text { Alvarenga } \\
\text { Cardoso Gimenes }\end{array}$ & $\begin{array}{c}\text { III conedu - } \\
\text { Congresso Nacional } \\
\text { de Educação }\end{array}$ & $\begin{array}{l}\text { A pesquisa de campo foi } \\
\text { realizada com quatro } \\
\text { alunos com deficiência } \\
\text { intelectual, que } \\
\text { frequentam as escolas da } \\
\text { rede municipal de } \\
\text { educação e trazem em } \\
\text { seu histórico a passagem } \\
\text { pela escola especial, e } \\
\text { com seus representantes. }\end{array}$ & $\begin{array}{l}\text { Discutir as principais } \\
\text { propostas da Política } \\
\text { Nacional de Educação } \\
\text { Especial na perspectiva da } \\
\text { Educação Inclusiva }\end{array}$ \\
\hline 6 & 2016 & $\begin{array}{c}\text { Microcefalia: } \\
\text { experiências e } \\
\text { expectativas junto à } \\
\text { Realidade materna }\end{array}$ & Nicolli Bertuoli & Monografia & $\begin{array}{l}\text { Mães de crianças que } \\
\text { nasceram com } \\
\text { microcefalia no segundo } \\
\text { semestre de } 2015 \text { na } \\
\text { Paraíba. }\end{array}$ & $\begin{array}{c}\text { Descrever as experiências de } \\
\text { mães de crianças com } \\
\text { microcefalia e suas } \\
\text { expectativas sobre o } \\
\text { desenvolvimento dos filhos. }\end{array}$ \\
\hline 7 & 2017 & $\begin{array}{c}\text { Crianças com } \\
\text { deficiência na } \\
\text { educação infantil: } \\
\text { caminhos para a } \\
\text { inclusão. }\end{array}$ & $\begin{array}{c}\text { André Fabiano } \\
\text { krüger }\end{array}$ & Monografia & $\begin{array}{l}\text { Professoras da Educação } \\
\text { Infantil da Rede } \\
\text { Municipal de Educação } \\
\text { de Florianópolis, Santa } \\
\text { Catarina e incluiu nove } \\
\text { participantes, todas } \\
\text { mulheres e professoras } \\
\text { atuantes na educação } \\
\text { infantil, em seis } \\
\text { diferentes unidades }\end{array}$ & $\begin{array}{l}\text { Analisar práticas } \\
\text { pedagógicas que } \\
\text { efetivamente contribuem à } \\
\text { inclusão escolar com } \\
\text { crianças com deficiências no } \\
\text { contexto da educação } \\
\text { infantil. }\end{array}$ \\
\hline
\end{tabular}




\section{Conclusões}

Para que os alunos com microcefalia possam ser englobados de forma eficaz, os educadores devem compreender a anomalia e desenvolver atividades que estimulem e/ou reabilitem os campos afetados como o neurológico, psíquico, motor, cognitivo e físico através de atividades específicas e peculiares. Dentre as práticas que facilitam a inclusão, o lúdico teve destaque, assim como o uso de materiais diversos, que despertam a curiosidade das crianças e possibilitam múltiplas experiências sensoriais.

\section{Referências}

BORDIN, Maria De Fatima Burger.Escola Inclusiva - Um Desafio Para o Instituto Federal Catarinense Campus Rio do Sul -SC, 2009.

BERODT, E. D. Microcefalia. Disponível em: <. Http: www.compartireducacionespecial.org>. Acesso em: 23 de dezembro de 2017.

FONSECA, Vitor D a. Educação especial: Programas de Estimulação Precoce - Uma Introdução às Idéias de Fuertein. 2 ed. rev.aum. Porto Alegre: artes Médicas, 1995.

FIOCRUZ, Fundação Oswaldo Cruz. Especiais Zika. . Disponível em: <.http: https://agencia.fiocruz.br/zika-0. Acesso em: 28 de dezembro de 2017.

HOWSTUFFWORKS B RASIL. "HowStuffWorks - O que é microcefalia?". Disponível em: <http://saude.hsw.uol.com.br/microcefalia.htm>. Consultado em: 28 de de dezembro de 2017.

LIBÂNEO. José Carlos. Pedagogia e pedagogos, para quê? 12. Ed. São Paulo: Cortez, 2010.

MARCELlI D.; COEH, D. Infância e Psicopatologia. $8^{a}$ ed. Porto Alegre: Artmed, 2010.

Ministério da Educação e Cultura (2002). Estratégias e orientações pedagógicas para a educação de crianças com necessidades educacionais especiais -Dificuldades acentuadas de aprendizagem. Deficiência múltipla. Brasília: MEC/SEE.

OLIVEIRA, S. M. (199 9). Valores e crenças de educadoras de creche sobre o desenvolvimento e educação de crianças de dois e três anos. Dissertação de Mestrado.

PAULON, Simone Mainieri; FREITAS, Lia Beatriz de Lucca; PINHO, Gerson Smiech. DOCUMENTO S UBSIDIÁRIO À POLÍTICA DE INCLUSÃO. Disponível em: 
<http://portal.mec.gov.br/seesp/arquivos/pdf/docsubsidiariopoliticadeinclusao.pdf $>$. Acesso em: 26 de dezembro de 2017.

SILVEIRA, Flávia Furtado; NEVES, Marisa Maria Brito da Justa. Inclusão Escolar de Crianças com Deficiência Múltipla: Concepções de Pais e Professores. Psicologia: Teoria e Pesquisa, Brasília, v. 22, n. 1, p.1-8, abr. 2006.

SÁ, Leila Maria Singh de Macedo Pinto e. Intervenção Precoce e Microcefalia Estratégias de Intervenção Eficazes, 2013.

VYGOTSKY, LEV S. A formação social da mente: o desenvolvimento dos processos psicológicos superiores. $4^{\text {a }}$.ed. São Paulo: Martins Fontes, 1991.

VELÁSQUEZ, F. R., Enfoques sobre el aprendizaje humano. Venezuela, 2001.

VYGOTSKY, LEV S. Pensamento e linguagem. São Paulo: Martins Fontes, 1987. 